\title{
Accuracy of Prognostic and Predictive Markers in Core Needle Breast Biopsies Compared with Excisional Specimens
}

\author{
Mohammad Motamedolshariati ${ }^{\mathrm{a}}$ Bahram Memar $^{\mathrm{b}}$ Mohsen Aliakbaian $^{\mathrm{b}}$ \\ Mohammad T. Shakeric Mohammad Samadi ${ }^{\mathrm{b}}$ Ali Jangjoo ${ }^{\mathrm{b}}$ \\ aVascular and Endovascular Surgery Research Center, Imam Reza Hospital, \\ 'Surgical Oncology Research Center, Imam Reza Hospital, \\ 'Department of Community Medicine, Faculty of Medicine, Mashhad University of Medical Sciences, Mashhad, Iran
}

\section{Keywords \\ Prognostic factors - Breast cancer - Core needle biopsy}

\section{Summary}

Background: Core needle biopsy (CNB) is widely accepted for preoperative diagnosis of breast cancer and sometimes can be the only way of providing a suitable specimen for prognostic and predictive marker studies prior to neoadjuvant treatment. The purpose of this study was to evaluate the accuracy of CNB by comparing histological tumor type and grade as well as estrogen receptor (ER), progesterone receptor (PR), p53, and HER2/ neu status by immunohistochemistry in CNB and excisional surgical specimens. Patients and Methods: During a 2.5-year study period, we identified 30 patients with breast cancer, who underwent CNB and definitive surgery. To evaluate the accuracy of CNB, tumor grade, ER, PR, HER2, and p53 status were immunohistochemically determined in both the CNB and the surgical specimens, and concordance of results between the 2 specimens was assessed. Results: The concordance rate was $100 \%$ for histological type, $66.6 \%$ for histological grade, and 96.7, 90, 76.7 and $93.3 \%$ for ER, PR, p53 and HER2/neu, respectively. Conclusion: Our study showed that CNB has an excellent accuracy for tumor type, ER, and HER2/ neu; however, it should be used cautiously for tumor grade, $\mathrm{PR}$, and p53 status. Thus, excisional biopsy is recommended for the determination of these factors.

\section{Introduction}

Percutaneous core needle biopsy (CNB) of breast masses is increasingly being used for obtaining a preoperative diagnosis $[1,2]$. This method may prevent unnecessary surgery, reduce costs, and is the only required diagnostic procedure in almost $90 \%$ of cases $[3,4]$. It is well documented that there is a good concordance rate between $\mathrm{CNB}$ and excisional biopsy for histological diagnosis of breast cancer [5, 6]. Additionally, CNB is increasingly relied on for providing information about tumor grade and different prognostic factors. This information is required for surgical planning and also for determining adjuvant systemic therapy, and can be obtained from CNB alone. There are few studies focusing on predictive and prognostic information obtained with CNB. According to various reports, estrogen receptor (ER) status, HER2/neu status, and histological type can be reliably assessed in CNB specimens [7-12]; however, there is significant discordance with regard to progesterone receptor (PR) $[13,14]$, histological grading [10], and p53 status [7, 13]. Therefore, this study was designed to determine the accuracy of percutaneous CNB through comparative analysis of histological tumor grade and type as well as ER, PR, HER2/neu, and p53 status in CNB and excisional biopsy.

\section{Patients and Methods}

The study was approved by the ethics committee of the Mashhad University of Medical Sciences. Between February 2009 and May 2011, all patients with breast masses suspicious for invasive breast cancer were included in this study in 2 different teaching hospitals. Informed consent was obtained from all participating patients. Preoperative CNB was performed under local anesthesia using 16-gauge automated core biopsy needles. At least 3 biopsies were obtained from each patient under clinical (in patients with superficial, palpable masses) or ultrasound guidance. The specimens were fixed in $10 \%$ formalin for $24 \mathrm{~h}$. After tissue processing and preparation of paraffin blocks, 3-micron paraffin sections were cut and sent off for pathologic and immunohistochemical (IHC) evaluation.

In this study, we prepared 3-micron sections with polylysine-coated slides for each marker. For IHC staining, slides were deparaffinized in xylene and graded ethyl alcohols and washed with distilled water. Then,

\section{KARGER}

Fax +497614520714

Information@Karger.com

www.karger.com
(C) 2014 S. Karger GmbH, Freiburg

$1661-3791 / 14 / 0092-0107 \$ 39.50 / 0$

Accessible online at:

www.karger.com/brc
Dr. Ali Jangjoo, MD

Surgical Oncology Research Cente

Imam Reza Hospital, Faculty of Medicin

Mashhad University of Medical Sciences

Mashhad, Iran

jangjooa@mums.ac.ir 
according to the DAKO (Glostrup, Denmark) protocol, slides were buffered with EDTA ( $\mathrm{pH}$ 6.5-7), and placed in a $100^{\circ} \mathrm{C}$ bain marie boiler for $30 \mathrm{~min}$. Endogenous peroxidase activity was quenched by treating slides with $3 \%$ hydrogen peroxide in methyl alcohol for $10 \mathrm{~min}$ and then with DAKO autoimmunostainer. The slides were incubated using a ChemMate $^{\mathrm{TM}}$ DAKO EnVision ${ }^{\mathrm{TM}}$ system (K5007) (with mouse monoclonal antibodies) for $45 \mathrm{~min}$ in $25^{\circ} \mathrm{C}$, and subsequently exposed to chromogen substrate for 1-2 min. Mayer's hematoxylin (Dako, S3301) was used to counterstain slides for $10-30 \mathrm{~min}$. Finally, lithium carbonate was added, and dehydration was done with ethyl alcohol.

In a total of 30 patients, breast cancer was identified in preoperative $\mathrm{CNB}$, and mastectomy was performed during the first month following CNB. Patients were excluded if they had received neoadjuvant chemotherapy or radiotherapy. The data evaluated were as follows: demographics, tumor grade and histology, ER, PR, HER2/neu, and p53 expression in both $\mathrm{CNB}$ and final excision specimens. Additionally, concordance rates were evaluated for the aforementioned data between CNB and final excision specimens.

Histological grade was determined based on pleomorphism, mitoses, and tubule formation [13]. ER and PR status were determined by IHC, and results were expressed as percentage of nuclear staining of tumor cells, in which $1 \%$ was defined as the cutoff point for positivity. We also determined p53 expression by IHC, and the cutoff point was defined in the same way.

According to the ASCO/USCAP guideline for HER2/neu evaluation, membranous staining was graded as negative (score 0 or 1 ), weakly positive or intermediate (score 2), and strongly positive (score 3 ). Hence, a score of 0 defined tumors with no staining or membrane staining in less than $10 \%$ of the tumor cells, and $1+$ defined tumors with faint membrane staining in more than $10 \%$ of the tumor cells (cell membranes partially stained). A weakly positive (intermediate) result was represented by a $2+$ score characterized by weak to moderate complete membrane staining in more than $10 \%$ of the tumor cells. Finally, $3+$ represented a strongly positive result which was defined as strong complete membrane staining in more than $10 \%$ of the tumor cells. In this study, we considered score $2+$ as positive. Complementary fluorescence in situ hybridization (FISH) was not used due to economic reasons and inaccessibility.
Normal breast tissue was considered as positive control for ER and PR and negative control for HER2, whereas ER- and PR-negative tumor tissue was regarded as negative control for ER and PR. HER2-positive tumor tissue was considered as positive control for HER2. Tumor tissue with negative and positive p53 expression was considered as negative and positive p53 control, respectively.

Sensitivity and specificity values were calculated by considering excisional biopsy as the gold standard and CNB as the test assessment. Kappa statistical analysis was used to measure the agreement between the 2 tests. Kappa values were defined as follows: 0 , none; $0-0.2$, slight; $0.2-0.4$, fair; 0.4-0.6, moderate; 0.6-0.8, substantial; and 0.8-1.0, complete [15].

\section{Results}

A total of 30 patients were included, in whom 25 had invasive ductal carcinoma, 4 had invasive lobular carcinoma, and 1 had apocrine carcinoma. The mean age was 51 years \pm standard deviation (SD) (range 32-88 years). The excised tumors were a mean $3.73 \mathrm{~cm} \pm \mathrm{SD}$ (range 1-13 cm) in diameter. The surgical procedure carried out was mastectomy in $25(83 \%)$ cases, while $5(17 \%)$ patients underwent breast-conserving surgery.

Sensitivity, specificity, concordance, and kappa values for CNB compared with surgical excision are presented in table 1, and sensitivity, specificity, and concordance are illustrated in figure 1. Comparison of histological examination revealed complete agreement between CNB and surgical specimens. Tumor grade was concordant in $20(66.6 \%)$ cases, with a kappa coefficient of 0.45 . The grade was lower in CNB than in the surgical specimens in $6(20 \%)$ cases and higher in $4(13.3 \%)$ cases (table 2). The ER status was the same in $29(96.7 \%)$
Table 1. Sensitivity, specificity, concordance, and kappa values for core needle biopsy (CNB) compared with surgical excision

\begin{tabular}{lllll}
\hline & Sensitivity, \% & Specificity, \% & Concordance, \% & Kappa value \\
\hline Histological type & - & - & 100.0 & 1.0 \\
Tumor grade & - & - & 66.6 & 0.45 \\
ER & 100.0 & 91.66 & 96.7 & 0.93 \\
PR & 93.7 & 85.7 & 90.0 & 0.79 \\
p53 & 70.58 & 84.21 & 76.7 & 0.537 \\
HER2/neu & 83.3 & 100.0 & 93.3 & 0.857 \\
\hline
\end{tabular}

$\mathrm{ER}=$ Estrogen receptor; $\mathrm{PR}=$ progesterone receptor.

Fig. 1. Bar chart of sensitivity, specificity, and concordance for estrogen receptor (ER), progesterone receptor (PR), HER2, and p53.

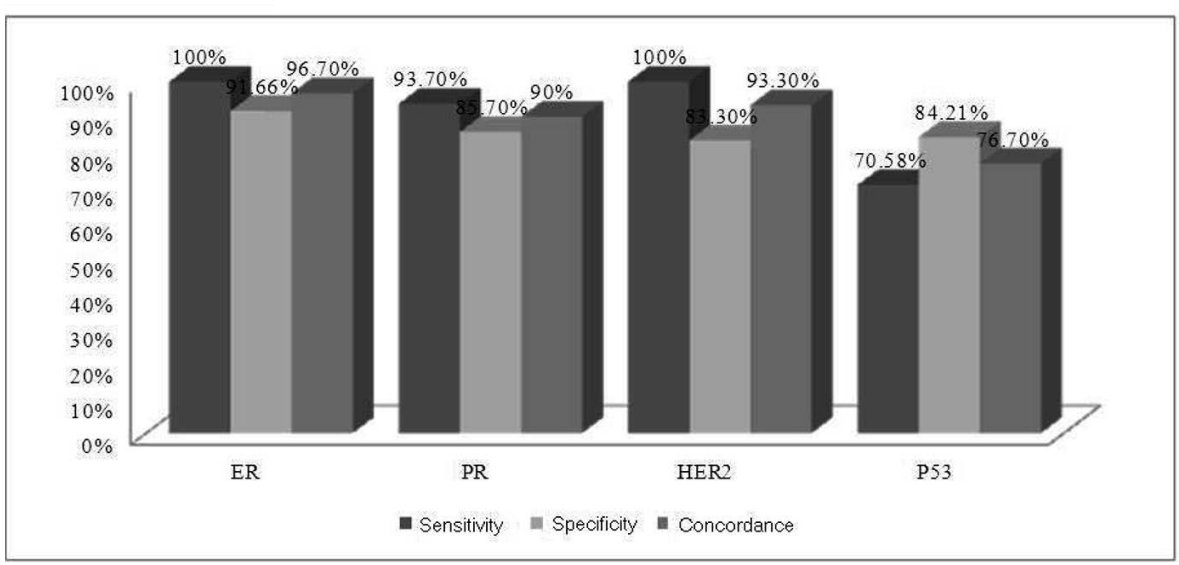


cases $($ kappa coefficient $=0.93$ ), which shows a good concordance rate between the 2 specimens (table 3 ). Discordance in ER assessment was detected in only 1 case in which a falsepositive result was obtained with the CNB specimen. With regard to PR status, discrepancy occurred in 3 cases, while the results were identical in $27(90 \%)$ cases (kappa coefficient $=$ 0.79) (table 3). In 2 of the 3 cases, PR was positive in CNB but negative in the final excisional biopsy. The third discrepancy was due to a false-negative result obtained with the CNB specimen. p53 was identical in 23 of 30 cases in both specimens. There was $76.7 \%$ agreement and a kappa coefficient of 0.53 (table 4). The observed discrepancies were the result of $2(6.6 \%)$ false-positive and 5 false-negative results $(16.6 \%)$ obtained with the CNB specimen. As for HER2/neu status, the result was the same in $28(93.3 \%)$ of 30 specimens (kappa coefficient $=0.857$ ), which shows good concordance (table 5). The score was false-negative in 2 cases $(6.6 \%)$ with $\mathrm{CNB}$, while there were no false-positive results $(0.0 \%)$.

\section{Conclusion}

Clinical TNM stage, pathological information, and molecular markers are used for making treatment decisions, and percutaneous $\mathrm{CNB}$ can provide adequately sized samples for this purpose [14]. However, the concern remains that CNB may be less reliable than excisional biopsy for providing information about various prognostic and predictive factors. This study was designed to assess the concordance of prognostic and predictive features and tumor marker expression in CNB compared to surgical excision.

In our study, we observed complete concordance between CNB and excisional biopsy for ER, and substantial concordance for PR. Other publications confirm CNB superiority in determining ER status. It is suggested that upscoring may occur in CNB compared with excisional biopsy, probably due

Table 2. Comparison of the histological grade in core needle biopsy (CNB) and surgical excision; values expressed as $\mathrm{n}(\%)$

\begin{tabular}{clrlr}
\hline & \multicolumn{3}{l}{ Surgical excision } \\
\cline { 2 - 5 } & +3 & +2 & +1 & total \\
\hline CNB & 6 & 3 & 0 & $9(30.0)$ \\
+1 & 1 & 11 & 3 & $15(50.0)$ \\
+2 & 1 & 3 & 3 & $6(20.0)$ \\
+3 & 0 & $17(56.6)$ & $6(20.0)$ & 30 \\
Total & $7(23.3)$ & & & \\
\hline
\end{tabular}

to better fixation in CNB than in surgical specimens [8], which was not confirmed in this study. Based on very good correlation $(96.7 \%)$, sensitivity (100\%), and specificity (91.66\%), ER can be accurately assessed in $\mathrm{CNB}$, which is in agreement with previous studies $[9,16]$.

Ough et al. [15] and Arnedos et al. [8] reported concordance rates of 78 and $85 \%$, respectively for PR, while this rate was $90 \%$ in our study. Furthermore, our results showed a higher sensitivity $(93.7 \%)$ and specificity (85.7\%). Although these rates may be reliable, further studies with larger sample sizes are required.

The level of agreement between $\mathrm{CNB}$ and excisional biopsy was $100 \%$ for histological typing and $66.6 \%$ for grading. Burge et al. [10] reported the same rate for typing but a higher rate for grading $(77 \%)$, while the rate for tumor type assessment was much lower (74\%) in the study by Harris et al. [11]. The disagreement with regard to histological typing is likely due to limited sample size and the use of tumor subclassifications which were not used in our study and in that of Burge et al. [10]. The low rate of agreement in terms of grading may be due to sampling errors.

CNB with early determination of the approximate status of prognostic factors can play a crucial role in early initiation of adjuvant treatment. Patients with tumors negative for both $\mathrm{ER}$ and PR are not considered for hormonal therapy, as the response would be less than $10 \%$. Tumors which are positive for both receptors will respond to this type of treatment in

Table 4. Comparison of p53 expression in core needle biopsy (CNB) and surgical excision; values expressed as n (\%)

\begin{tabular}{llll}
\hline & \multicolumn{2}{l}{ Surgical excision } & \\
\cline { 2 - 4 } & positive & negative & total \\
\hline CNB & 12 & 2 & $14(46.6)$ \\
Positive & 5 & 11 & $16(53.3)$ \\
Negative & $17(56.6)$ & $13(43.3)$ & 30 \\
Total & & & \\
\hline
\end{tabular}

Table 5. Comparison of HER2/neu status in core needle biopsy (CNB) and surgical excision; values expressed as n (\%)

\begin{tabular}{|c|c|c|c|c|c|}
\hline & Surgical e & ision & & & \\
\hline & 0 & +1 & +2 & +3 & total \\
\hline $\mathrm{CNB}$ & & & & & \\
\hline 0 & $8(26.6)$ & $2(6.66)$ & $1(3.33)$ & 0 & $11(36.6)$ \\
\hline+1 & 5 (16.6) & $3(10.0)$ & $1(3.33)$ & 0 & $9(30.0)$ \\
\hline+2 & 0 & 0 & $4(13.33)$ & $1(3.33)$ & 5 (16.6) \\
\hline+3 & 0 & 0 & $1(3.33)$ & $4(13.33)$ & 5 (16.6) \\
\hline Total & $13(43.3)$ & 5 (16.6) & $7(23.3)$ & $5(16.6)$ & 30 \\
\hline
\end{tabular}

Table 3. Comparison of estrogen receptor $(\mathrm{ER})$ and progesterone receptor $(\mathrm{PR})$ in core needle biopsy (CNB) and surgical excision; values expressed as $n(\%)$

\begin{tabular}{|c|c|c|c|c|c|c|}
\hline & \multicolumn{6}{|c|}{ Surgical excision } \\
\hline & \multicolumn{3}{|l|}{ ER } & \multicolumn{3}{|l|}{ PR } \\
\hline & positive & negative & total & positive & negative & total \\
\hline \multicolumn{7}{|l|}{$\mathrm{CNB}$} \\
\hline Positive & 18 & 1 & $19(63.3)$ & 15 & 2 & $17(56.6)$ \\
\hline Negative & 0 & 11 & $11(36.7)$ & 1 & 12 & $13(43.3)$ \\
\hline Total & $18(60.0)$ & $12(40.0)$ & 30 & $16(53.3)$ & $14(46.6)$ & 30 \\
\hline
\end{tabular}


more than $50 \%$ of cases, and a response of $33 \%$ will be achieved in those positive for 1 receptor [17].

HER2/neu assessment showed good concordance between CNB and surgical excision in this study (93.3\%). There were only 2 cases with false-negative results, and no false-positive results were seen. According to our data, $\mathrm{CNB}$ is reliable for determining the HER2/neu status. Other studies have reported similar concordance rates of $80-92 \%$ [10, 12]. However, it is recommended that CNB specimens with edge or retraction artifacts should not be used for this purpose [18]. Patients with HER2-positive cancers having no adjuvant therapy will have a poorer prognosis. This indicates that these genes are of prognostic significance. HER2 positivity determines the rate of response to anthracycline treatment and taxane-based therapy. Considering that treatment with nonanthracycline- and non-taxane-based therapy will be less useful, HER2-positive tumors often become resistant to endocrine therapy [19].

For p53, the concordance rate was $76.7 \%$ in our study, which is similar to the results of Ough et al. [15]. Other studies have reported concordance rates of $85-100 \%$ [7].

The limitation of this study is the small sample size which prohibits generalization of the results. In conclusion, our study suggests that ER and HER2/neu status can be accurately assessed in CNB. The high discordance rate for tumor grading, $\mathrm{PR}$, and p53 in CNB specimens should be interpreted with caution. However, it is recommended to perform complementary studies using FISH and chromogenic in situ hybridization (CISH) tests.

\section{Acknowledgment}

The results described in this paper formed part of an MD thesis submitted by the 4th author to the Mashhad University of Medical Sciences. The study was supported by the Vice Chancellor for Research, Mashhad University of Medical Sciences. We gratefully acknowledge Ms. M. Hassanpour for editing the manuscript.

\section{Disclosure Statement}

The authors declare no conflict of interest.

\section{References}

1 Benson SR, Harrison NJ, Lengyel J, Deacon C, Isgar B: Combined image guidance excision of non-palpable breast lesions. Breast 2004;13:110-4.

2 Verkooijen HM, Peeters PH, Pijnappel RM, Koot VC, Schipper ME, Borel Rinkes IH: Diagnostic accuracy of needle-localized open breast biopsy for impalpable breast disease. Br J Surg 2000;87:344-7.

3 White RR, Halperin TJ, Olson JA Jr, Soo MS, Bentley RC, Seigler HF: Impact of core-needle breast biopsy on the surgical management of mammographic abnormalities. Ann Surg 2001;233:76977.

4 Rubin E, Mennemeyer ST, Desmond RA, Urist MM, Waterbor J, Heslin MJ, Bernreuter WK, Dempsey PJ, Pile NS, Rodgers WH: Reducing the cost of diagnosis of breast carcinoma: impact of ultrasound and imaging-guided biopsies on a clinical breast practice. Cancer 2001;91:324-32.

$\checkmark 5$ Dillon MF, Hill AD, Quinn CM, O'Doherty A, McDermott EW, O'Higgins N: The accuracy of ultrasound, stereotactic, and clinical core biopsies in the diagnosis of breast cancer, with an analysis of false-negative cases. Ann Surg 2005;242:701-7.

6 Verkooijen HM: Diagnostic accuracy of stereotactic large-core needle biopsy for nonpalpable breast disease: results of a multicenter prospective study with $95 \%$ surgical confirmation. Int J Cancer 2002; 99:853-9.

7 Rakha EA, Ellis IO: An overview of assessment of prognostic and predictive factors in breast cancer needle core biopsy specimens. J Clin Pathol 2007; 60:1300-6.
-8 Arnedos M, Nerurkar A, Osin P, A'Hern R, Smith IE, Dowsett M: Discordance between core needle biopsy (CNB) and excisional biopsy (EB) for estrogen receptor (ER), progesterone receptor $(\mathrm{PgR})$ and HER2 status in early breast cancer (EBC). Ann Oncol 2009;20:1948-52.

9 Lorgis V, Algros MP, Villanueva C, Chaigneau L, Thierry-Vuillemin A, Nguyen T, Demarchi M, Bazan F, Sautiere JL, Maisonnette-Lescot Y, Ringenbach F, Bontemps P, Pivot X: Discordance in early breast cancer for tumour grade, estrogen receptor, progesterone receptors and human epidermal receptor-2 status between core needle biopsy and surgical excisional primary tumour. Breast 2011;20:284-7.

10 Burge CN, Chang HR, Apple SK: Do the histologic features and results of breast cancer biomarker studies differ between core biopsy and surgical excision specimens? Breast 2006;15:167-72.

11 Harris GC, Denley HE, Pinder SE, Lee AH, Ellis IO, Elston CW, Evans A: Correlation of histologic prognostic factors in core biopsies and therapeutic excisions of invasive breast carcinoma. Am J Surg Pathol 2003;27:11-5.

12 Mann GB, Fahey VD, Feleppa F, Buchanan MR: Reliance on hormone receptor assays of surgical specimens may compromise outcome in patients with breast cancer. J Clin Oncol 2005;23:5148-54.

13 Elston CW, Ellis IO: Pathological prognostic factors in breast cancer. I. The value of histological grade in breast cancer: experience from a large study with long-term follow-up. Histopathology 1991;19:403-10.
14 Pijnappel RM, van Dalen A, Borel Rinkes IH, van den Tweel JG, Mali WP: The diagnostic accuracy of core biopsy in palpable and non-palpable breast lesions. Eur J Radiol 1997;24:120-3.

15 Ough M, Velasco J, Hieken TJ: A comparative analysis of core needle biopsy and final excision for breast cancer: histology and marker expression. Am J Surg 2011;201:692-4.

16 Usami S, Moriya T, Amari M, Suzuki A, Ishida T, Sasano H, Ohuchi N: Reliability of prognostic factors in breast carcinoma determined by core needle biopsy. Jpn J Clin Oncol 2007;37:250-5.

17 Hunt KK, Newman LA, Copeland III EM, Blad KI: The breast; in Brunicardi F (ed) Schwartz's Principles of Surgery. New York, NY McGraw-Hill, 2010, p. 453.

18 Wolff AC, Hammond ME, Schwartz JN, Hagerty KL, Allred DC, Cote RJ, Dowsett M, Fitzgibbons PL, Hanna WM, Langer A, McShane LM, Paik S, Pegram MD, Perez EA, Press MF, Rhodes A, Sturgeon C, Taube SE, Tubbs R, Vance GH, van de Vijver M, Wheeler TM, Hayes DF: American Society of Clinical Oncology/ College of American Pathologists guideline recommendations for human epidermal growth factor receptor 2 testing in breast cancer. Arch Pathol Lab Med 2007;131:18-43.

19 Bhargava R, Esposito NN, Dabbs DJ: Diagnostic immunohistochemistry of breast; in Dabbs DJ (ed): Diagnostic Immunohistochemistry. Philadelphia, PA, Churchill Livingstone Elsevier, 2010, p. 797. 